\title{
Gerencia transformacional desde el accionar docente directivo para el abordaje del Contexto Escolar
}

\section{Transformational management from the managing teacher directive for the approach of the School Context}

\author{
Carmen Morgado \\ Carmen5morgado@gmail.com \\ Liceo Nacional Antonio Maceo \\ Grupo de Investigación CIENCIAMATRIA \\ Julio Juvenal Aldana Zavala \\ julioaldanazavala@gmail.com \\ Universidad Nacional Experimental Francisco de Miranda \\ Grupo de Investigación CIENCIAMATRIA \\ https://orcid.org/0000-0002-7934-9103 \\ Josía isea \\ josiaisea@gmail.com \\ Universidad Nacional Experimental Francisco de Miranda \\ Grupo de Investigación CIENCIAMATRIA \\ https://orcid.org/0000-0001-8921-6446
}

Recibido: $18 / 08 / 2017$

Aprobado: 27/10/2017 


\section{RESUMEN}

El objetivo de la investigación es Analizar la gerencia transformacional desde el accionar docente directivo para el abordaje del contexto escolar del Liceo Nacional Antonio Maceo, Las Dos Bocas, municipio Colina, estado Falcón. Metodológicamente está suscrita en el paradigma positivista, tipo de campo, nivel descriptivo, diseño no experimental, la población y la muestra son 52 personas del colectivo institucional, la técnica e instrumento de recolección de datos fue la encuesta y el cuestionario, el análisis de los datos fue a través de la estadística descriptiva. Luego de aplicar el instrumento se concluye que si no se maneja una gerencia que motive e impulse otras formas de ver la educación, simplemente se seguirá en solitario educando a un grupo de estudiantes donde sus aprendizajes no serán cónsonos con lo que se exige desde la comunidad y la familia. La alianza estratégica entre familia, escuela y comunidad debe iniciarse en la gerente a través de un liderazgo transformacional que permita una intervención e integración para beneficio de todos, especialmente cuando se hace referencia a un medio rural, donde la atención de todos es primordial.

Palabras clave: gerencia transformacional, accionar docente directivo, contexto escolar.

\section{SUMMARY}

The objective of the research is to analyze the transformational management from the directive teaching actions to address the school context of Antonio Maceo National High School, Las Dos Bocas, Colina municipality, Falcón state. Methodologically it is subscribed in the positivist paradigm, type of field, descriptive level, non-experimental design, the population and the sample are 52 people from the institutional collective, the technique and instrument of data collection was the survey and the questionnaire, the analysis of the data was through descriptive statistics. After applying the instrument, it is concluded that if a management is not managed that motivates and promotes other ways of viewing education, it will simply continue on its own, educating a group of students where their learning will not be consonant with what is demanded from the community. and the family. The strategic alliance between family, school and community must begin in the manager through a transformational leadership that allows an intervention and integration for the benefit of all, especially when referring to a rural environment, where the attention of all is paramount.

Key words: transformational management, directive teaching actions, school context. 


\section{CIENCIAMATRIA \\ Revista Interdisciplinaria de Humanidades, Educación, Ciencia y Tecnología \\ Año V. Vol. V. №9. Julio - Diciembre 2019 \\ Hecho el depósito de ley: pp201602FA4721 \\ ISSN-L: 2542-3029; ISSN: 2610-802X \\ Universidad Nacional Experimental Francisco de Miranda (UNEFM). Santa Ana de Coro. Venezuela}

Carmen Morgado; Julio Juvenal Aldana Zavala; Josía isea

\section{INTRODUCCIÓN}

Los cambios sociales que se han venido generando en los últimos años han intervenido en el desempeño de las organizaciones, siendo las escuelas una de las más afectadas, pues cada una de ellas obedece a las particularidades del contexto comunitario donde está inmersa, así como del contexto escolar conformado por el colectivo institucional, padres, representantes, estudiantes, fuerzas vivas y otras organizaciones comunitarias. En virtud de ello, la actividad profesional de directivos y docentes, debe tomar un vuelco para enfrentar las complejidades e incertidumbres desde sus espacios, bien sea la escuela en su totalidad o el aula de clases, sin embargo, es necesario acotar que cada uno de estos escenarios está conformado a su vez por un conjunto de conocimientos, procedimientos, actitudes, valores, prácticas y principios que se consolidan según las particularidades e individualidades de estos procesos.

Entonces, dicha consolidación solo es posible a través de la práctica eficiente de la gerencia transformacional, como un factor clave para el personal directivo y docente, y que les permitirá adaptarse a los nuevos paradigmas que emergen desde la sociedad.

Al respecto, (Quintero y Marrero, 2013), plantean preocupación cuando afirman:

Los procesos educativos y la gestión de las organizaciones escolares deben experimentar cambios fundamentales, replantear sus enfoques y sus métodos, superar el paradigma lineal y construir nuevas formas de producir y adquirir conocimiento. Una propuesta educativa, fundamentada en una Perspectiva Sistémica, impulsará procesos inéditos de humanización, alejada de prácticas que violenten o victimicen a los actores vinculados en la aventura de aprender para la vida y vivir aprendiendo (p. 136).

En función de lo expresado por los autores, las escuelas requieren de un personal directivo que no solo se prepare académicamente, sino que además tenga vocación de servicio, principios y valores para el trabajo exigente, que fomente el trabajo en equipo, busque una comunicación efectiva y asertiva con todos, enfocarse a estrategias y acciones que no solo permitan la formación de los estudiantes, sino además la consolidación de un equipo de trabajo de calidad y una comunidad educativa 


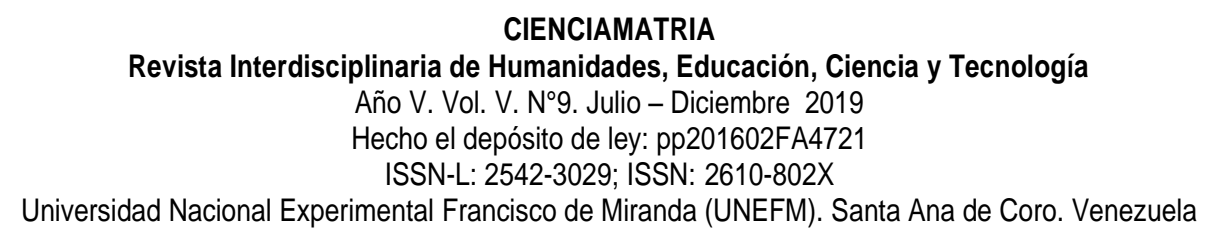

Carmen Morgado; Julio Juvenal Aldana Zavala; Josía isea

acompañando dicho proceso escolar.

Cuando se hace referencia a la gerencia educativa, implica los principios básicos de la administración y la gestión desde la comunidad educativa, pues una escuela es una organización social que administrativamente obedece a los postulados gerenciales en cualquier escenario. Hablar de gerencia desde la escuela, abarca un aspecto que está dirigido hacia la calidad y la excelencia, no solo de los procesos educativos que allí emergen, sino además de las prácticas administrativas, de planeación, organizativas, de control y evaluación dirigidas hacia el contexto escolar donde está inserta la escuela. Para (Gerstner, 1996), la gerencia transformacional implica: "un proceso de influir sobre las personas para dirigir sus esfuerzos hacia el cumplimiento de determinadas metas, lo cual va a depender del estilo personal y las exigencias de la situación" (p. 15), cuando se hace referencia a este tipo de gerencia, se vincula con la transfiguración de la gente y las organizaciones, ubicar actuaciones y acciones que busquen la innovación como una forma de pensar y sentir para direccionar los procesos técnicos, administrativos y pedagógicos.

Algunos autores consideran que gerencia y liderazgo son sinónimo, en ese sentido, (Mosley, 2005), argumenta que el liderazgo transformacional: "es un cambio de paradigma hacia un estilo más visionario y más enfocado en la dotación de facultades, el cual es necesario en un mundo de cambios" (p. 9). La gerencia desde esta vertiente transformacional implica dejar a un lado el tradicionalismo de la administración para enfocarse hacia una perspectiva más novedosa que responda a las exigencias de la organización y de la sociedad.

Al respecto, (Núñez, 2002) considera seis características básicas de los directivos como líderes gerenciales: "habilidad mental, madurez emocional, necesidad intrínseca de logro, habilidad para resolver problemas, empatía y representatividad", (p. 3). En vista de ello, la gerencia basada en un liderazgo transformacional, desde el escenario educativo, requiere un trabajo en equipo, una visión compartida entre todos los 


\section{Carmen Morgado; Julio Juvenal Aldana Zavala; Josía isea}

involucrados, emprenderse positivamente y canalizar las incertidumbres hacia el logro efectivo de los objetivos institucionales.

Por lo tanto, una gerencia transformacional desde la vertiente educativa, implica un personal educativo directivo con habilidades y destrezas comunicativas, mediador, motivador, adaptarse y transcender a las situaciones inesperadas en el día a día de la convivencia escolar, saber actuar en condiciones de incertidumbre, vincularse con el contexto inmediato, tanto dentro como fuera de la institución, sobre todo enfocar su gestión hacia la participación e integración de todos los actores educativos en pro de la formación de los estudiantes y el mejoramiento de la calidad de vida, así como de la educación como proceso transformacional de las personas.

En virtud de ello, cuando se relaciona la gerencia desde el contexto escolar, no es más que la participación de todos los involucrados en el hecho educativo en el mejoramiento y búsqueda de los procesos de eficacia, eficiencia y calidad que deben generarse desde la escuela. El contexto escolar está conformado por los estudiantes y el colectivo institucional: directivo, administrativo, docente, obrero, padres, representantes y estudiantes, cada uno cumple con un rol primordial para que la organización socio educativa logre las metas y objetivos que se establecen desde el inicio del año escolar. Afirmar que solo la gerencia hará posible el buen desempeño de la institución educativa, es negar que se requiere del trabajo en equipo para gestionar, administrar y enfocar todos los esfuerzos administrativos, sociales, pedagógicos, en beneficio de los estudiantes. En tal sentido, no se niega la responsabilidad del gerente educativo, sin embargo, el necesita de un apoyo permanente del equipo de trabajo, lo cual dependerá de la motivación, actitud y organización que lleve a cabo con el talento humano que le rodea para propiciar los conocimientos, procedimientos y actitudes de enfocar el hecho educativo hacia la calidad y la excelencia. En complemento de lo planteado, para (Schavino, 2012),

Es totalmente válido que el personal directivo como líder que oriente las acciones de su equipo de trabajo y colaboradores, adopte y ejecute 


\section{Carmen Morgado; Julio Juvenal Aldana Zavala; Josía isea}

estrategias gerenciales en procura de resultados notorios dentro de las instituciones que dirige, porque, en el contexto educativo, permitirían la sistematización de las acciones con el fin de lograr el adecuado funcionamiento organizacional de la escuela $y$, en consecuencia, el aprendizaje del estudiantado, eventos que a la larga serán el apoyo para el desarrollo del país. (p. 13)

Desde esta perspectiva, es a través de la gerencia que se abre el abanico de posibilidades y decisiones encaminadas hacia la visión y misión institucional, así como las demandas y exigencias del contexto que rodea a la escuela. (Ohmae, 2005) respalda esta perspectiva, al señalar que:

Es por medio de las estrategias gerenciales, como los entes directivos pueden identificar las realidades del contexto escolar en función de las fortalezas, oportunidades, debilidades o amenazas; así como establecer prioridades con el fin de diseñar planes para utilizar los recursos de manera eficiente; también coordinar, ejecutar y controlar las acciones para finalmente evaluar resultados y re planificar las tareas en procura de lograr los objetivos. (p. 10)

De acuerdo a lo referido por el autor, la gerencia necesita vincularse con las nuevas tendencias, entre ellas la planteada en esta investigación, como lo es la gerencia transformacional, para complementar la administración, organización, del colectivo de la escuela, hacia una gestión educativa de excelencia, siempre y cuando se tome en cuenta el enfoque humanista que debe prevalecer desde la gerencia y la administración, sobre esa base generar el más alto rendimiento individual y colectivo.

En relación a la gerencia desde el ámbito escolar, (Alfonso, 2001), señala "quien enfrenta hoy el reto de dirigir una institución educativa, más que un administrador tendrá necesariamente que ser un líder educativo, para lograr resultados óptimos en las condiciones en que vivimos" (p. 13); ser director de una escuela no solo es cumplir con los requisitos para ese cargo, implica una trayectoria docente y directiva enfocada hacia la productividad, una visión gerencial de liderazgo transformacional para todos los participantes en el hecho educativo. 


\section{Carmen Morgado; Julio Juvenal Aldana Zavala; Josía isea}

En la actualidad en Venezuela, la gerencia educativa es un tema de suma importancia que se enfoca hacia la calidad en eficiencia administrativa, pedagógica y social, esto dependerá de la relación armónica con el contexto escolar, buscando compromiso, pertinencia, trabajo mancomunado, dirección hacia un mismo norte fundamentado en la visión, misión y objetivos establecido para el hecho educativo.

Por lo que es pertinente señalar lo que plantea (Bolívar, 2010): "la capacidad para mejorar de un centro escolar depende, de manera relevante, de equipos directivos con liderazgo que contribuyan a dinamizar, apoyar y animar que aprenda a desarrollarse, contribuyendo a construir la capacidad interna de mejora" (p. 3). Solo el trabajo desde la gerencia transformacional cambiará las vertientes hacia donde se dirige la escuela, el trabajo pedagógico y la unión entre todos los involucrados.

Desde la perspectiva del problema de investigación, en el Liceo Nacional Antonio Maceo ubicado en la comunidad de Las Dos Bocas, parroquia Acurigua, municipio Colina, estado Falcón, Venezuela, se observan algunas debilidades desde la gerencia de esta institución educativa, que enfocan la necesidad de promover una gerencia transformacional para el abordaje del contexto escolar, estas debilidades son caracterizadas por: falta de vinculación entre la dirección del plantel y la comunidad que rodea la escuela, deficiente comunicación entre el personal directivo y los colectivos docentes, administrativos y obreros, poca integración y participación de los padres y representantes con la institución, escaso apoyo de las organizaciones comunitarias hacia el Liceo.

Desde este contexto, se organizan grillas cultuales y deportivas, se evidencia a través de observaciones que la asistencia de los padres y miembros de la comunidad es muy poca, ante una situación irregular dentro de la institución no se cuenta con el apoyo de los padres y la comunidad, existe una apatía con énfasis hacia lo que significa la institución educativa con amplia trascendencia en la comunidad, existen algunos padres que nunca han visitado la institución, ni siquiera para inscribir o retirar boletines. 


\section{CIENCIAMATRIA \\ Revista Interdisciplinaria de Humanidades, Educación, Ciencia y Tecnología \\ Año V. Vol. V. №9. Julio - Diciembre 2019 \\ Hecho el depósito de ley: pp201602FA4721 \\ ISSN-L: 2542-3029; ISSN: 2610-802X \\ Universidad Nacional Experimental Francisco de Miranda (UNEFM). Santa Ana de Coro. Venezuela}

\section{Carmen Morgado; Julio Juvenal Aldana Zavala; Josía isea}

Asimismo, se evidencia un exceso de trabajo administrativo por parte del director disminuyendo su atención hacia el personal docente, no se formulan metas y objetivos institucionales ni al principio ni durante del año escolar, apatía por parte de los miembros de la escuela y de la comunidad hacia esta. Ante ello, el personal directivo tiende a verbalizar expresiones negativas hacia el personal dejando a un lado el respeto, en ocasiones el gerente se siente aislado y solo al querer el apoyo del personal, la rutina pedagógica y administrativa se ha fomentado cada día, generando apatía dentro de la propia institución, y haciéndose notar hacia la comunidad.

Esta problemática puede generarse a causas como desvinculación entre el director y el resto de la comunidad educativa (personal, organizaciones comunitarias, otros), prácticas gerenciales obsoletas a la complejidad social de la actualidad, desinterés por parte de la dirección en vincularse con la comunidad, actitud errada de la dirección para involucrar a las familias y comunidad en el hecho educativo, carencia de objetivos y metas institucionales predefinidos.

Asimismo, entre las consecuencias de la problemática poca integración y participación de las familias y la comunidad a la institución educativa, falta de trabajo mancomunado con la sociedad, procesos pedagógicos desvirtuados de la realidad social donde se inserta la escuela, estilo gerencial inadecuado, entre otras. Complementando lo planteado, (Gutiérrez, Rodríguez e Isea, 2017: 232), plantean que "Por lo tanto, si se sigue con este modelo pedagógico no se podrá dar respuesta oportuna a las necesidades sociales, ni mucho menos a las exigencias del mercado laboral actual".

Siendo pertinente generar un nuevo modelo o enfoque de abordar la gerencia en el contexto educativo, con la finalidad de promover la sinergia necesaria para contribuir en trabajar desde lo gerencial, en el fomento de una educación transformadora de la persona, hacia la consecución de una ciudadanía crítica, reflexiva, protagónica, basada desde el aprender a lo largo de la vida, para lo cual se requiere la cooperación del equipo de trabajo de la organización educativa. 


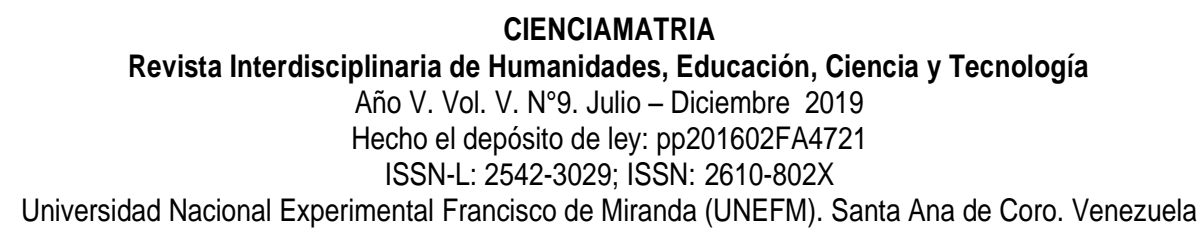

Carmen Morgado; Julio Juvenal Aldana Zavala; Josía isea

Desde lo planteado, se genera la siguiente interrogante: ¿Cómo se desarrolla la gerencia transformacional desde el accionar docente directivo para el abordaje del contexto escolar del Liceo Nacional Antonio Maceo, Las Dos Bocas, municipio Colina, estado Falcón?

\section{Objetivo de la investigación}

Analizar la gerencia transformacional desde el accionar docente directivo para el abordaje del contexto escolar del Liceo Nacional Antonio Maceo, Las Dos Bocas, municipio Colina, estado Falcón.

\section{REFERENCIAL TEÓRICO}

\section{Gerencia Transformacional}

La gerencia transformacional es influir, transformar, cambiar a las personas y sus organizaciones en virtud de sus sentimientos, pensamientos y acciones. De allí que el liderazgo asuma una posición importante para lograr nuevas percepciones del deber ser, la idea es que puedan generarse cambios radicales que sean congruentes con los espacios y donde la motivación sea un factor clave para ello.

Por consiguiente, (Gerstner, 1996) se refiere al liderazgo como: "proceso de influir sobre las personas para dirigir sus esfuerzos hacia el cumplimiento de determinadas metas, lo cual va a depender del estilo personal y las exigencias de la situación" (p. 56), este estilo personal, que se suma al profesional implica una serie de elementos que conforman el perfil del gerente, los cuales se convierten en sus características para ejercer esa influencia en el resto de las personas.

Al respecto, (Núñez, 2002) considera seis características básicas de los directivos como líderes gerenciales, entre ellas: "habilidad mental, madurez emocional, necesidad intrínseca de logro, habilidad para resolver problemas, empatía y representatividad" ( $p$. 3). Cuando el autor plantea la habilidad mental, es el grado de inteligencia, el cual debe 


\section{CIENCIAMATRIA \\ Revista Interdisciplinaria de Humanidades, Educación, Ciencia y Tecnología \\ Año V. Vol. V. №9. Julio - Diciembre 2019 \\ Hecho el depósito de ley: pp201602FA4721 \\ ISSN-L: 2542-3029; ISSN: 2610-802X \\ Universidad Nacional Experimental Francisco de Miranda (UNEFM). Santa Ana de Coro. Venezuela}

\section{Carmen Morgado; Julio Juvenal Aldana Zavala; Josía isea}

ser superior, al del resto de las personas que conforman la organización, así como el dominio de habilidades y destrezas acordes a las funciones de líder y gerente que debe aplicar, las cuales son esenciales desde el rol que cumple en la institución, es decir, es un estrategia y dinamizador de los procesos para hacerlos más cónsonos a lo que el momento amerita y exige.

Asimismo, la madurez emocional es la confianza que tienen los líderes y gerentes en ellos mismos, en sus actuaciones, convicciones, que los hace ser asertivos ante las respuestas que la organización exige por situaciones de incertidumbre, esta madurez emocional le permite asumir con compromisos las fallas y consecuencias de todas sus actuaciones desde la gerencia. Por otra parte, la necesidad intrínseca de logro, es ese interés o impulso interno que lo lleva a cumplir con su función en virtud del logro exigido desde su cargo, a través del diseño de metas y el establecimiento de objetivos y propósitos de acuerdo a las exigencias de su función gerencial las cuales deben cumplirse.

Del mismo modo, la habilidad para resolver problemas, donde diferencia las causas de los efectos y centran su atención y actuación hacia las causas para gestionar las estrategias y recursos necesarios para atacarlas y emprender procesos positivos a su gestión. Otra característica es la empatía, como la habilidad de los gerentes para identificarse emocionalmente con los demás, poniéndose en los zapatos de cada uno de ellos y buscando soluciones y actuaciones cónsonas con las circunstancias.

Como ultima característica, la representatividad, implica la actuación desde sus funciones con el rol de gerente, directivo y líder. Todas estas características antes descritas deben ser asumidas con efectividad desde quien ocupa la gerencia como una forma de garantizar una gestión de excelencia hacia cada uno de sus subordinados y hacia la organización como tal, asumiendo además el compromiso que se tiene con la comunidad donde está inserta la escuela y cada una de las familias que conforman la comunidad educativa. 


\section{CIENCIAMATRIA \\ Revista Interdisciplinaria de Humanidades, Educación, Ciencia y Tecnología \\ Año V. Vol. V. №9. Julio - Diciembre 2019 \\ Hecho el depósito de ley: pp201602FA4721 \\ ISSN-L: 2542-3029; ISSN: 2610-802X \\ Universidad Nacional Experimental Francisco de Miranda (UNEFM). Santa Ana de Coro. Venezuela}

\section{Carmen Morgado; Julio Juvenal Aldana Zavala; Josía isea}

En este sentido, el gerente transformacional debe desarrollar habilidades y destrezas comunicativas para una permanente interacción con su personal, las familias y los miembros de la comunidad, convirtiéndose en mediador ante las diversas situaciones que se generan y afecten a la escuela, como espacio primario de su accionar; además debe ser fuente de motivación para cada uno de los que participa en el hecho educativo en miras de cumplir con las exigencias sociales, dar respuestas asertivas a las condiciones de incertidumbre, y saber afrontar aquellos episodios que perjudiquen el buen desempeño organizacional.

Cabe destacar, que su accionar gerencial debe vincularse en su totalidad con el acto educativo, el mejoramiento de la calidad de vida, el compromiso con los cambios socios educativos, la participación e integración de todos los que conforman el conglomerado escolar, la formación integral de los estudiantes. Este liderazgo transformacional es dirigido hacia los parámetros democráticos que se suscitan desde la sociedad, pues la educación se corresponde con las políticas de estado que se generen desde los escenarios micros de gobernabilidad.

Al respecto, para que un liderazgo sea más positivo al colectivo, (Bolívar, 2004) señala: "los integrantes del grupo deben ser corresponsables a la hora de decidir sobre la persona en la que va a recaer el liderazgo, los términos a ejercer, las condiciones a cumplir tanto por el líder como por los corresponsables" (p. 13); los integrantes del grupo deben ser líderes para garantizar el liderazgo de forma efectiva.

De igual manera, (Mosley, 2005) argumenta que: "el liderazgo transformacional es un cambio de paradigma hacia un estilo más visionario y enfocado en la dotación de facultades, el cual es necesario en un mundo de cambios" (p. 28). Este autor plantea, además, que existen tres factores que definen al gerente transformacional, como son: liderazgo carismático, consideración individualizada y estimulación intelectual. Cuando hace referencia al liderazgo carismático, son las características que definen la personalidad de líder, hace énfasis en el respeto, orgullo, espíritu de grupo, centra su 


\section{CIENCIAMATRIA \\ Revista Interdisciplinaria de Humanidades, Educación, Ciencia y Tecnología \\ Año V. Vol. V. No9. Julio - Diciembre 2019 \\ Hecho el depósito de ley: pp201602FA4721 \\ ISSN-L: 2542-3029; ISSN: 2610-802X \\ Universidad Nacional Experimental Francisco de Miranda (UNEFM). Santa Ana de Coro. Venezuela}

\section{Carmen Morgado; Julio Juvenal Aldana Zavala; Josía isea}

atención a lo importante e indispensable, le da sentido a la visión, misión, de la organización.

El segundo factor es la consideración individualizada, es la delegación de asignaciones para que los demás aprendan dando una atención especial a los individuos. El tercer factor es la estimulación intelectual, a través de la visión como gerente y la generación de ideas novedosas sobre gerencia atendiendo a estrategias y acciones oportunas. Estos factores son necesarios desde la gerencia educativa porque se lograrán mejoras, propiciará el trabajo en equipo, aumentará la eficiencia, ayuda a que otros sean líderes.

\section{Comunicación y gerencia transformacional}

La comunicación interpersonal es una condición necesaria para el ejercicio del liderazgo transformacional, el gerente desde esta vertiente debe ser un comunicador eficaz no solo desde la dimensión personal, sino además profesional permitiéndole asumir cualidades y competencias acordes con los objetivos organizacionales que desea lograr con su equipo de trabajo, y es a través de la comunicación que lo puede alcanzar. Para (Rodríguez, 1993) expresa que la comunicación: "es la esencia misma de la vida del hombre, no se puede vivir sin ella y en sus constantes intercambios, para ello es necesario utilizar tres niveles de comunicación, la intrapersonal, la interpersonal y la social" (p. 23).

En palabras de este autor, la comunicación le permite comunicarse consigo mismo, con los demás y con un auditorio o publico en específicos. La comunicación es necesaria para la participación, la toma de decisiones, se hace necesaria para la práctica de un liderazgo efectivo. El citado autor destaca: "que sin una buena comunicación los líderes no pueden lograr motivar a sus seguidores" (p. 4); sin un proceso comunicativo efectivo no se logra consolidar una organización desde la gerencia.

Desde esta perspectiva, la comunicación es una herramienta para un liderazgo transformacional efectivo, considerada un elemento estratégico de ella, gracias a los 


\section{Carmen Morgado; Julio Juvenal Aldana Zavala; Josía isea}

flujos de información y comunicación que se genera en la organización. Desde los ambientes escolares y educativos, la comunicación permite estimular las relaciones entre los diferentes colectivos de la escuela, las personas de la comunidad y los miembros de las familias, es la esencia de una relación efectiva, y de allí parten otras acciones cónsonas con las funciones de la comunicación.

Al respecto, (Pasquali, 2003) define la comunicación como "la relación comunitaria humana, consistente en la emisión - recepción de mensajes entre interlocutores en estado de total reciprocidad, siendo por ello un factor esencial de convivencia y un elemento determinante de las formas que asume la sociabilidad del hombre" (p. 51). Un proceso comunicativo es una condición para que interactúen efectivamente dos o más personas logrando comprensión, entendimiento, de las informaciones que se comparten.

Entre el gerente y las demás personas debe existir una comunicación. Implica que le permitan llevar a cabo los procesos administrativos de forma armónica, como son: planificación, organización, control y evaluación; todo ello dirigido hacia la consolidación del liderazgo transformacional directivo. En este sentido, la comunicación abierta entre los colectivos institucionales, familiares y comunitarios debe ser una condición necesaria para facilitar la transformación educativa a través de diálogos, conversaciones, donde las ideas, los sentimientos, se discutan, se logren acuerdos, consenso, para el beneficio de todos.

La buena comunicación permite el intercambio mutuo de ideas para unificar criterios y lograr el éxito de la gerencia transformacional efectiva. Asimismo, la condición es inherente a la condición humana a través de la cual se logra una relación comunicativa humana, convivencia, socialización que le permiten a la organización consolidar el liderazgo transformacional por medio de cambios, apertura, participación y compromiso colectivo; además para la toma de decisiones.

Cabe destacar, que la comunicación es un elemento importante para la escuela porque 


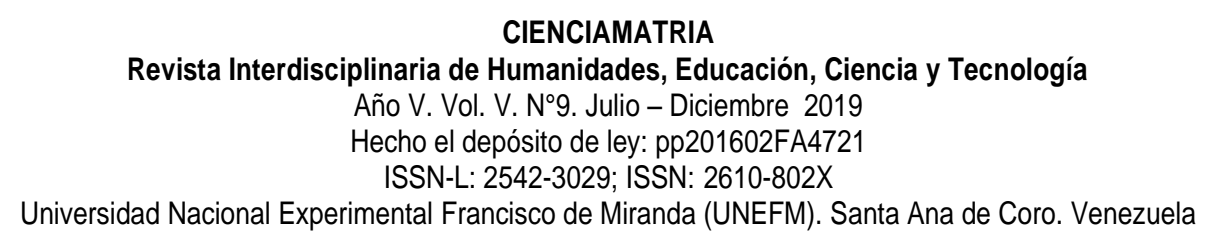

Carmen Morgado; Julio Juvenal Aldana Zavala; Josía isea

a través de ella se generan relaciones interpersonales entre todos sus integrantes, incluyendo a los estudiantes, sin embargo ella es necesaria desde la gerencia y sus relaciones con las familias y la comunidad para darle soluciones a las diversas problemáticas y consolidar una trasformación educativa desde la gerencia, a través de un liderazgo comunicativo efectivo.

Según (Kelinger, 1998), los aspectos claves en la comunicación interpersonal son: el desarrollo de relaciones (ayuda y coordinación de acciones para el logro de los objetivos institucionales), reciprocidad en la comunicación (los comportamientos de unos dependen de otros), influencia y comunicación (compromiso para cumplir las actividades, incentivos y compensaciones para lograr las metas), comunicación terapéutica ( ayudar a entender a sí mismo y con los demás), empatía (comprensión de los sentimientos de la otra persona y actuar en virtud de ello), honestidad (comunicación veraz, franca y sincera), validación (respeto y aceptación de los demás), cuidado (indicación emocional que se expresa entre comunicadores).

\section{Motivación y gerencia transformacional}

La importancia de la motivación en la organización y desde la gerencia es como mantener el entusiasmo de los trabajadores para el logro de las metas, siendo esta una condición importante para todo líder transformacional. En este sentido, (Robbins, 1993), define la motivación como: "el deseo de hacer mucho esfuerzo por alcanzar las metas de la organización, condicionado por la posibilidad de satisfacer alguna necesidad individual" (p. 89), en palabras del autor la motivación es el empeño e impulso con el que se hacen as acciones para conseguir las metas. Este es un proceso dinámico que busca la satisfacción de las necesidades y lograr sentimientos gratificantes.

\section{Toma de decisiones y gerencia transformacional}

La calidad educativa depende de las acciones que se hayan tomado desde una toma de decisiones asertivas y validas de acuerdo a las circunstancias. Por consiguiente, 


\section{CIENCIAMATRIA \\ Revista Interdisciplinaria de Humanidades, Educación, Ciencia y Tecnología \\ Año V. Vol. V. №9. Julio - Diciembre 2019 \\ Hecho el depósito de ley: pp201602FA4721 \\ ISSN-L: 2542-3029; ISSN: 2610-802X \\ Universidad Nacional Experimental Francisco de Miranda (UNEFM). Santa Ana de Coro. Venezuela}

\section{Carmen Morgado; Julio Juvenal Aldana Zavala; Josía isea}

(Melinkoff, 2001) define el proceso de toma de decisiones como: "la facultad de escoger la mejor y más conveniente alternativa de un conjunto posible de ellas" (p. 6), las decisiones se toman a través de técnicas muy específicas, y desde el liderazgo transformacional, estas son válidas, como son: consenso, expresión de ideas, lluvia de ideas, opiniones, soluciones, otras.

Para llegar al consenso, dicho autor recomienda: (a) considerar todos los aspectos de la situación; (b) analizar las objeciones a las diversas alternativas de solución; (c) aprovechar las diferencias de opinión bien intencionadas para obtener información adicional; (d) aclarar dudas; y (e) obligar al grupo a buscar mejores soluciones. La toma de decisiones desde el conceso amerita el monitoreo y seguimiento constante para que se logren las pautas para las cuales se generaron las decisiones. El líder transformacional debe buscar el conceso permanente entre la familia, escuela y comunidad con la idea de buscar soluciones efectivas, responsabilidad, aprovechamiento del tiempo y un ambiente agradable.

La toma de decisiones le permite al gerente transformacional analizar las opciones, identificar lo que se debe hacer, buscar los criterios para actuar en función de las decisiones, evaluar las alternativas, establecer los riesgos, seleccionar la mejor decisión. La toma de decisiones no solo requiere de un trabajo gerencial, sino además del desarrollo de un líder que influya en los demás y se logren tomas de decisiones efectivas de acuerdo a las circunstancias.

\section{METODOLOGÍA DE LA INVESTIGACIÓN}

Se considera una Investigación tipo de campo, de acuerdo con (Palella y Martins, 2010) "consiste en la recolección de datos directamente de la realidad donde ocurren los hechos, sin manipular o controlar las variables. Estudia los fenómenos sociales en su ambiente natural" (p. 38). La información necesaria para realizar la investigación fue recogida directamente del colectivo institucional. 


\section{Carmen Morgado; Julio Juvenal Aldana Zavala; Josía isea}

La investigación se apoyó en un nivel de carácter descriptivo, teniendo como referencia el apoyo de (Chávez, 2010) quien expresa que una "investigación descriptiva consiste en la caracterización de un hecho, fenómeno o grupo con el fin de establecer su estructura o comportamiento" (p. 46). Se concibe como descriptiva debido a que se describió las condiciones y el grado del accionar docente directivo para el abordaje del contexto escolar del Liceo Nacional Antonio Maceo, Las Dos Bocas, municipio Colina, estado Falcón.

Considerando que el diseño obedece a la estrategia que adopta el investigador para responder al problema, y en función del tipo de investigación propuesto, se tiene un diseño no experimental, que los autores (Palella y Martins, 2010) lo refieren como: "el que se realiza sin manipular en forma deliberada ninguna variable" (p. 82). Es no experimental porque no se manipularon las variables de la investigación, solo se redujo a observar y medir el objeto de estudio tal como se originó en su contexto natural, para después analizarlos. Para el caso de la investigación, el diseño no experimental es de tipo transversal, pues se analizaron las variables en un único momento.

En cuanto a la población de estudio. (Chávez, 2010), plantea que esta es "un conjunto finito o infinito de elementos con características comunes para los cuales serán extensivas las conclusiones de la investigación" (p. 81). Para obtener la información necesaria se consideró como población el colectivo institucional del Liceo Nacional Antonio Maceo. La población para este estudio estuvo constituida por 52 personas que conforman el colectivo institucional, siendo esta de carácter finita y manejable por los investigadores, por lo que se procedió al abordaje investigativo de la totalidad de la población señalada.

El uso de las técnicas de recolección de datos permite obtener las informaciones o datos necesarios para la investigación de diferentes maneras. En esta investigación se hizo uso de la técnica de la encuesta, en este sentido, (Palella y Martins, 2010) refiere que: "la encuesta es una técnica destinada a obtener datos de varias personas cuyas 
opiniones interesan al investigador" (p. 111). Por consiguiente, la encuesta es una técnica que permite obtener informaciones y opiniones a las que le parece atractivo al investigador, requiere de fases como: preparación, diseño, ejecución, análisis y presentación de los resultados.

El instrumento de recolección de datos en esta investigación, fue el cuestionario; el cual fue diseñado por los investigadores para tal fin y estuvo dirigido a las 52 personas que conforman la población de estudio, el instrumento constó de 14 preguntas con una escala de estimación con las alternativas: Siempre, casi siempre, algunas veces, casi nunca y nunca. Para (Palella y Martins, 2010): "el cuestionario es un instrumento de investigación que forma parte de la técnica de la encuesta" (p. 119).

Para analizar los resultados obtenidos en la recolección de los datos se utilizó la estadística descriptiva, por cuanto se presentaron los resultados en tablas de frecuencias y gráficos, lo que posteriormente permitió el análisis de los mismos, (Palella y Martins, 2010) determinan que este tipo de estadística: "se refiere a la recolección, presentación, descripción, análisis e interpretación de una colección de datos, esencialmente consiste en resumir éstos con uno o dos elementos de información (medidas descriptivas) que caracterizan la totalidad de los mismos" (p. 89).

\section{ANÁLISIS DE LOS RESULTADOS}

De acuerdo con el objetivo de investigación y las variables de estudio, se muestran a continuación un resumen de los resultados obtenidos de la aplicación del instrumento a la población en estudio, en el cual se midieron las dimensiones de la gerencia transformacional y el contexto escolar. 


\section{Cuadro 1.}

Respuestas del Ítem El personal directivo posee necesidades intrínsecas hacia logros institucionales

\begin{tabular}{lrr}
\multicolumn{1}{c}{ Opciones } & Frecuencia & \multicolumn{2}{c}{ Porcentaje } \\
\hline Siempre & - & - \\
Casi siempre & - & - \\
Algunas veces & - & $8 \%$ \\
Casi nunca & 4 & $92 \%$ \\
Nunca & 48 & $100 \%$ \\
\hline \multicolumn{1}{c}{ Total } & 52 & \\
\hline
\end{tabular}

El $92 \%$ de los encuestados considera que nunca el personal directivo posee necesidades intrínsecas hacia logros institucionales, así como el 8\% opina que casi nunca lo hace. Las necesidades intrínsecas le permiten a cualquier persona auto motivarse para alcanzar metas, si el directivo no busca razones para mejorar su gestión, la escuela decae progresivamente. En este sentido, (Núñez,2002) considera que la necesidad intrínseca de logro, es ese interés o impulso interno que lo lleva a cumplir con su función en virtud del logro exigido desde su cargo, a través del diseño de metas y el establecimiento de objetivos y propósitos de acuerdo a las exigencias de su función gerencial las cuales deben cumplirse.

Es de suma importancia que se apliquen estrategias basadas en la gerencia transformacional, con la finalidad de promover aptitudes en la comunidad docente y directiva de la institución que permitan constituir equipos de trabajo, basados desde la motivación intrínseca, lo cual permitirá gestionar un trabajo cooperativo en transcender las adversidades que se puedan presentar, así como gestionar una visión para trabajar con pensamiento complejo y efectivo. 


\section{Cuadro 2.}

Respuestas del Ítem El personal directivo es empático

\begin{tabular}{lrr}
\multicolumn{1}{c}{ Opciones } & Frecuencia & \multicolumn{2}{c}{ Porcentaje } \\
\hline Siempre & - & - \\
Casi siempre & 12 & $23 \%$ \\
Algunas veces & 23 & $44 \%$ \\
Casi nunca & 17 & $33 \%$ \\
Nunca & - & - \\
\multicolumn{1}{c}{ Total } & 52 & $100 \%$ \\
\hline
\end{tabular}

El $44 \%$ de los encuestados considera que algunas veces el personal directivo es empático, seguido de un $33 \%$ que opina que casi nunca lo es, y un $23 \%$ con la opción casi siempre. La empatía es una cualidad personal que debe ser adquirida por las personas que laboran con público, en este caso, el personal educativo debe buscar la empatía como un medio de fortalecer los lazos afectivos y relaciones interpersonales para convivir en un ambiente de armonía. (Núñez, 2002) señala que la empatía, es la habilidad de los gerentes para identificarse emocionalmente con los demás, poniéndose en los zapatos de cada uno de ellos y buscando soluciones y actuaciones cónsonas con las circunstancias.

La empatía es una aptitud necesaria en el gerente educativo, por cuanto esta permite generar relaciones interpersonales basadas en el humanismo, en el reconocimiento del otro como persona, en la medida que el otro también hace lo propio. Puede señalarse que la empatía permite concebir acciones que fortalecen los equipos de trabajos en su cooperación, no debe confundirse empatía con ser un gerente permisivo, sino, que desde la empatía se puede conocer las necesidades e intereses de las demás personas, situación que puede ser aprovechada favorablemente para la generación de estratégicas gerenciales transformacionales que permitan articular situaciones basadas en el ganar - ganar en el cumplimiento de metas, objetivos, basados en un enfoque de calidad y efectividad. 


\section{Cuadro 3.}

Respuestas del Ítem El personal directivo motiva al colectivo institucional hacia el accionar con el contexto escolar

\begin{tabular}{lrr}
\multicolumn{1}{c}{ Opciones } & Frecuencia & Porcentaje \\
\hline Siempre & - & - \\
Casi siempre & - & - \\
Algunas veces & 12 & $23 \%$ \\
Casi nunca & 38 & $73 \%$ \\
Nunca & 2 & $4 \%$ \\
\multicolumn{1}{c}{ Total } & 52 & $100 \%$ \\
\hline
\end{tabular}

El $73 \%$ de los encuestados considera que casi nunca el personal directivo motiva al colectivo institucional hacia el accionar con el contexto escolar, seguido de un $23 \%$ con la opción algunas veces y un $4 \%$ con nunca. La motivación es un elemento clave desde la gerencia y el liderazgo, su práctica propicia el trabajo en equipo y el sentido de pertenencia. (Robbins, 1993), define la motivación como: "el deseo de hacer mucho esfuerzo por alcanzar las metas de la organización, condicionado por la posibilidad de satisfacer alguna necesidad individual" (p. 89), la motivación es el empeño e impulso con el que se hacen as acciones para conseguir las metas. Este es un proceso dinámico que busca la satisfacción de las necesidades y lograr sentimientos gratificantes.

Un gerente educativo en primer lugar debe estar motivado, desde allí podrá generar la motivación pertinente en el equipo de trabajo, relación que permitirá articular una relación recíproca, armónica, sinérgica, en procura de fomentar una labor educativa pensada y trabajada con eficacia, humanismo, con la finalidad de cumplir no solo metas, sino de transcender como organización hacia la consolidación de una educación que permee en la formación de un ciudadano con visión integral, en función de estar en consonancia con las exigencias de una sociedad del conocimiento, donde se requiere de personas que aprendan e investiguen a lo largo de la vida. 


\section{Cuadro 4.}

Respuestas del Ítem Se siente responsable como docente de la integración entre la familia, la escuela y la comunidad.

\begin{tabular}{lrr}
\multicolumn{1}{c}{ Opciones } & Frecuencia & Porcentaje \\
\hline Siempre & 6 & $11 \%$ \\
Casi siempre & 32 & $62 \%$ \\
Algunas veces & 14 & $27 \%$ \\
Casi nunca & - & - \\
Nunca & - & - \\
\multicolumn{1}{c}{ Total } & 52 & $100 \%$ \\
\hline
\end{tabular}

El 62\% de los encuestados consideran que los docentes se sienten responsables de la integración entre la familia, escuela y comunidad, seguido de un $27 \%$ algunas veces y un $11 \%$ siempre. El docente se asume esta responsabilidad por el contacto directo que tienen con los estudiantes, de allí que manifiesta que la integración y su efectividad es un compromiso adquirido desde su profesional. Para (Guedez, 2009) "la gestión educativa debe ser permanente que transcienda las paredes de la escuela y la vincule a la vida comunitaria y a los medios de comunicación" (p. 67), la sociedad es un espacio en la cual sumado a la familia y la escuela actúen en beneficio y cumplimiento de las políticas educativas, donde la participación sea propicia no solo para las actividades académicas, sino además culturales, deportivas, entre otras.

Una política educativa que se maneje desde la gerencia transformacional, es la de integración de la escuela con la comunidad inherente a esta, son diversos las proposiciones que se han realizado sobre el tema, pero es pertinente generar una acción directiva que permita diseñar planes estratégicos donde la comunidad se perciba como parte de la escuela, siendo así que podría promoverse la construcción de un recinto escolar productivo, sinérgico, efectivo, en donde la comunidad se vea parte del aprendizaje generado en la escuela, siendo así, una oportunidad para transitar en el aprendizaje a lo largo de la vida propuesto desde la sociedad del conocimiento. 


\section{CIENCIAMATRIA \\ Revista Interdisciplinaria de Humanidades, Educación, Ciencia y Tecnología \\ Año V. Vol. V. №9. Julio - Diciembre 2019 \\ Hecho el depósito de ley: pp201602FA4721 \\ ISSN-L: 2542-3029; ISSN: 2610-802X \\ Universidad Nacional Experimental Francisco de Miranda (UNEFM). Santa Ana de Coro. Venezuela}

Carmen Morgado; Julio Juvenal Aldana Zavala; Josía isea

\section{CONCLUSIONES}

Los estilos gerenciales llevados a cabo en el Liceo Nacional Antonio Maceo, se caracterizan por no ser transformacionales, hay muy poca influencia positiva con el colectivo institucional, se carece de motivaciones intrínsecas para diseñar y ejecutar acciones dirigidas hacia logros institucionales, la efectividad en la resolución de problemas es muy poca, siendo una de sus causas la falta de toma de decisiones participativa y democrática. La empatía y la comunicación interpersonal son débiles.

Todo este panorama institucional a través de la gerencia es preocupante, desde los episodios cambiantes en que se está sumergido en la actualidad, donde se reclama una gerencia proactiva, dinámica, enfocada en cada uno de los miembros que conforma el colectivo de la escuela, pero también en función de los intereses personales de cada uno, que deben perfilarse hacia una intervención positiva y efectiva de la comunidad y la familia como aliados inseparables de la escuela.

Si no se maneja una gerencia que motive e impulse otras formas de ver la educación, simplemente se seguirá en solitario educando a un grupo de estudiantes donde sus aprendizajes no serán cónsonos con lo que se exige desde la comunidad y la familia. La alianza estratégica entre familia, escuela y comunidad debe iniciarse en la gerente a través de un liderazgo transformacional que permita una intervención e integración para beneficio de todos, especialmente cuando se hace referencia a un medio rural, donde la atención de todos es primordial.

Dentro de este orden de ideas, el accionar de la gerencia con los colectivos institucionales y comunitarios, se manifiesta a través de falta de motivación hacia las instituciones que son parte del Liceo porque conforman el sistema donde está inserto. Tanto la comunidad como la familia, son instituciones sociales que colaboran con la escuela para una mayor efectividad en beneficio de los estudiantes; una escuela no puede funcionar sola, del mismo modo, la familia y las instituciones de la comunidad 


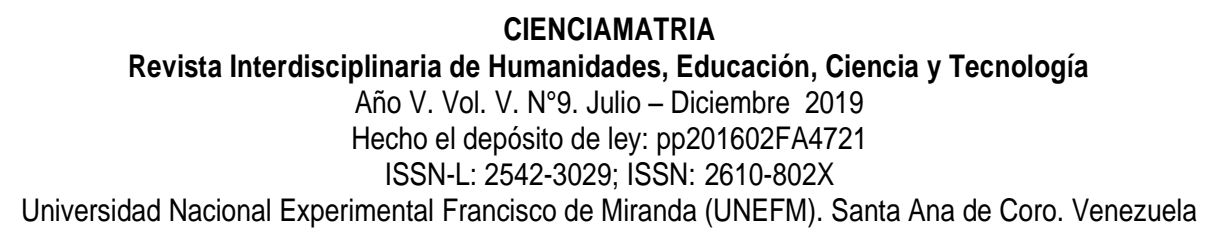

Carmen Morgado; Julio Juvenal Aldana Zavala; Josía isea

como consejos comunales, iglesias, comercios, otras escuelas, entre otras, necesitan de ella para educar a sus habitantes.

Todo este marco sistema conformado por escuela, familia y comunidad amerita de una gerencia escolar con un fuerte liderazgo para formar colectivos efectivos en virtud del beneficio de todos. Solo una gerencia comprometida con la escuela puede realizar acciones que promuevan el trabajo cooperativo y en equipo para evaluar las fortalezas y debilidades y generara planes de acción que vinculen a la institución educativa con el resto de las organizaciones para intervenir efectivamente,

Por último, las características de la gerencia transformacional para el abordaje del contexto escolar, deben ser bajo condiciones dinámicas, activas, proactivas, equilibradas y creativas para mejorar aquellas dificultades que se presentan en la escuela, así como saber qué hacer ante las circunstancias de incertidumbre. Una gerencia transformacional busca la integración escolar con compromiso y responsabilidad de todos los miembros del colectivo, buscar alianzas y estrategias basadas en la comunicación efectiva, toma de decisiones participativa, la integración escolar es asumida con un compromiso y responsabilidad exclusiva de los docentes

\section{REFERENCIAS CONSULTADAS}

1. Alfonso A. (2001). La excelencia gerencial en educación. Lima: Derrama Magisterial. (Biblioteca Pública de Lima).

2. Bolívar, A. (2004). Liderazgo Educativo y Reestructuración Escolar. Recuperado de htpp: dewey uab.es/pmarques/dioe/DOELiderazgo.doc.htm.

3. Bolívar A. (2010). El liderazgo educativo y su papel en la mejora: una revisión actual de sus posibilidades y limitaciones. Psicoperspectivas. Individuo y Sociedad, Vol. 9, No. 2. España: Universidad de Granada. 


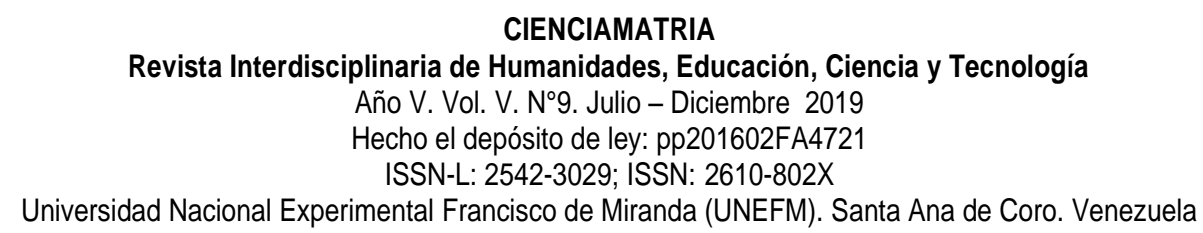

Carmen Morgado; Julio Juvenal Aldana Zavala; Josía isea

4. Chávez, N (2010). Introducción a la Investigación Educativa. Maracaibo Estado Zulia.

5. Gerstner .J. (1996). Reinventando La Educación. Barcelona. Piados.

6. Gutiérrez, N., Rodríguez, N., \& Isea, J. (2017). La emocionalidad frente a la innovación curricular. Revista Arbitrada Interdisciplinaria Koinonía, 2(3), 226-246. Recuperado de http://fundacionkoinonia.com.ve/ojs/index.php/revistakoinonia/article/view/61/48

7. Kelinger, F. (1998). Un enfoque conceptual en investigación del comportamiento. Recuperado de: www.uv.es/aidipe/planes/metoduleonMetDisTec.htm

8. Melinkoff, R. (2001). Los Procesos Administrativos. Caracas. Venezuela. Editorial. Panapo.

9. Mosley, D. (2005) Supervisión, la práctica del empowerment, Desarrollo de Equipos de Trabajo y su Motivación. México 6aㅡ Edición. Editores Internacional Thomson.

10.Núñez (2002), Modelo de Gerencia Educativa Bajo un Enfoque de Liderazgo Compartido para Mejorar la Estructura Organizacional en Escuelas Básicas. Trabajo de Grado. Universidad Pedagógica Experimental Libertador. Barquisimeto.

11.Ohmae, K. (2005). El próximo escenario global. Desafíos y oportunidades en un mundo sin fronteras. Bogotá: Editorial Norma.

12.Palella, S. y Martins, F. (2010). Metodología de la Investigación Cuantitativa. Ediciones FEDEUPEL. 2da Edición. Caracas Venezuela.

13.Pascuali, (2003). El Liderazgo. Recuperado de htpp: deweyuab.es/ pmarques/dioe/DOE Liderazgo.doc.htm

14. Quintero, E, y Marrero, E. (2013). Análisis Cibernético, Holístico y Transformacional en la Organización Escolar. Un Manual para el Director. Administración Educacional Anuario del Sistema de Educación en Venezuela /Año 1 - N 1. [Revista en línea] Universidad de los Andes (ULA). Mérida. 


\section{CIENCIAMATRIA}

Revista Interdisciplinaria de Humanidades, Educación, Ciencia y Tecnología

Año V. Vol. V. №9. Julio - Diciembre 2019

Hecho el depósito de ley: pp201602FA4721

ISSN-L: 2542-3029; ISSN: 2610-802X

Universidad Nacional Experimental Francisco de Miranda (UNEFM). Santa Ana de Coro. Venezuela

Carmen Morgado; Julio Juvenal Aldana Zavala; Josía isea

15. Robbins, S. (1993). Comportamiento Organizacional. México. Mc Graw-Hill.

16. Rodríguez C. (1993). El nuevo escenario. La cultura de la calidad y la productividad en las empresas. México: Editorial Trillas.

17.Schavino, N. (2012). La complementariedad gerencial en contextos organizacionales complejos. Revista Honoris Causa, 3, 11-12.

C2019 por los autores. Este artículo es de acceso abierto y distribuido según los términos y condiciones de la licencia Creative Commons Atribución-NoComercial-Compartirlgual 4.0 Internacional (CC BY-NC-SA 4.0) (https://creativecommons.org/licenses/by-nc-sa/4.0/). 